\title{
Kaon Condensation in Neutron Stars
}

Angels Ramos ${ }^{1}$, Jürgen Schaffner-Bielich ${ }^{2}$, and Jochen Wambach ${ }^{3}$

I Departament d'Estructura i Constituents de la Matèria, Universitat de Barcelona, Diagonal 647, 08028 Barcelona, Spain

2 RIKEN BNL Research Center, Brookhaven National Laboratory, Upton, New York 11973-5000, USA

3 Institut für Kernphysik, TU Darmstadt, Schlossgartenstr. 9, 64289 Darmstadt, Germany

\begin{abstract}
We discuss the kaon-nucleon interaction and its consequences for the change of the properties of the kaon in the medium. The onset of kaon condensation in neutron stars under various scenarios as well its effects for neutron star properties are reviewed.
\end{abstract}

\section{Introduction - hadrons in dense matter}

Due to its non-abelian structure, Quantum Chromodynamics (QCD) becomes very strongly interacting and highly nonlinear at large space-time distances. As a consequence, quarks and gluons condense in the physical vacuum with a gain in condensation energy density of $\Delta \epsilon_{0} \sim 500 \mathrm{MeV} / \mathrm{fm}^{3}$. The condensation of quarks is associated with the spontaneous breaking of chiral symmetry, an additional symmetry of the QCD Lagrangian in the absence of (current) quark masses. This limit is well justified in the up-down quark sector and to a somewhat lesser extent for the strange quark. There is good evidence that the mechanism for spontaneous chiral symmetry breaking is provided by classical gluon field configurations in euclidean space called 'instantons'. These provide effective quark-(anti)quark interactions which are strong enough to cause a BCS like transition to a condensed state of quarks and antiquarks. It has been shown, that this picture provides an excellent description of hadronic states and correlation functions [1] and it is fair to say that the low-lying hadron spectrum is dominated by spontaneous chiral symmetry breaking with confinement playing a much lesser role. These observations form the basis for discussing the properties of hadrons, or more precisely hadronic correlation functions, under extreme conditions in temperature and/or density as encountered in the early universe or in the interior of neutron stars. It is well established from lattice QCD that the vacuum undergoes a phase transition (or at least a sharp cross over) when heated. Chiral symmetry is restored in this phase accompanied by a nearly vanishing chiral quark condensate. Though not calculable at present from first principles, the same is expected to happen at finite density. Since light hadrons are dynamically driven by chiral symmetry breaking it is obvious to ask how hadronic properties are related to the vacuum structure and its changes with temperature and density. This is far from trivial and under intense experimental and theoretical scrutiny at present. 
As detailed below the most economical way for treating hadrons in matter under extreme conditions is to resort to 'effective field theories' in which hadrons rather than quarks and gluons appear as the fundamental degrees of freedom. Formally one identifies the pertinent quark currents $J_{\Gamma_{j}}(x)=\bar{q}(x) \Gamma_{j} q(x), \Gamma_{j}=$ $1, \gamma_{5}, \gamma_{\mu}, .$. with elementary hadronic fields $\phi_{i}(x)$ for which the most general effective Lagrangian, consistent with the underlying symmetries and anomaly structure of QCD, is written down. We recall that the spontaneous breaking of chiral symmetry has two important consequences. One is the appearance of (nearly) massless Goldstone bosons (pions, kaons, eta) and the other the absence of parity doublets in the hadron spectrum $\left(m_{\pi} \neq m_{f_{0}}, m_{\rho} \neq m_{a_{2}}\right.$ etc). For the present discussion the first is the most relevant. Chiral symmetry does more than just predict the existence of Goldstone bosons. It also prescribes and severely restricts their mutual interactions as well as those with other hadrons. The most rigorous treatment is in terms of 'chiral perturbation theory' [2] but other 'chiral effective Lagrangians' including e.g. vector mesons can be devised [3,4].

To elucidate the connection between the vacuum structure (the chiral condensate) and the properties of light hadrons let us consider a medium of hadronic matter in thermal and chemical equilibrium. This is of course well suited for neutron stars. The QCD partition function is then given in the grand-canonical ensemble as

$$
\mathcal{Z}_{Q C D}\left(V, T, \mu_{q}\right)=\operatorname{Tr} \exp \left\{-\left(\hat{H}_{Q C D}-\mu_{q} \hat{N}_{q}\right) / T\right\},
$$

where $\hat{H}_{Q C D}$ denotes the QCD Hamiltonian, $\hat{N}_{q}$ the quark number operator and $\mu_{q}$ the quark chemical potential. Statistical expectation of operators are then given as

$$
\langle\langle\hat{O}\rangle\rangle=Z^{-1} \sum_{n}\langle n|\hat{O}| n\rangle \exp \left\{-\left(E_{n}-\mu_{q} N_{n}\right) / T\right\},
$$

where $E_{n}$ are the exact QCD energies (hadrons). The quark condensate $\langle\langle\bar{q} q\rangle\rangle$ can be obtained directly from the free energy density

$$
\Omega_{Q C D}(T, V)=-\lim _{V \rightarrow \infty} \frac{T}{V} \ln \mathcal{Z}_{Q C D}\left(V, T, \mu_{q}\right)
$$

via the Feynman-Hellmann theorem as

$$
\langle\langle\bar{q} q\rangle\rangle=\frac{\partial \Omega_{Q C D}}{\partial m_{q}^{\circ}},
$$

where $m_{q}^{0}$ denotes the bare (current) quark mass. An obvious first step is to approximate the free energy density by an ideal gas of hadrons. Using the GellMann Oakes Renner relation for the vacuum chiral condensate

$$
m_{\pi}^{2} f_{\pi}^{2}=-2 m_{q}^{\circ}\langle\bar{q} q\rangle,
$$

where $m_{\pi}$ is the pion mass and $f_{\pi}$ the pion weak-decay constant, one then finds

$$
\frac{\langle\langle\bar{q} q\rangle\rangle}{\langle\bar{q} q\rangle}=1-\sum_{h} \frac{\Sigma_{h} \varrho_{h}^{s}\left(\mu_{q}, T\right)}{f_{\pi}^{2} m_{\pi}^{2}}
$$


Here

$$
\Sigma_{h}=m_{q}^{\circ} \frac{\partial m_{h}}{\partial m_{h}^{0}}
$$

denotes the 'sigma' commutator' (related to the scalar density of quarks in a given hadron) and $m_{h}$ the vacuum mass of a given hadron. At low temperature and small baryochemical potential $\left(\mu_{B}=3 \mu_{q}\right)$, in which the hadron gas is dominated by thermally excited pions and a free Fermi gas of nucleons, (6) leads to the celebrated leading-order result

$$
\frac{\langle\langle\bar{q} q\rangle\rangle}{\langle\bar{q} q\rangle}=1-\frac{T^{2}}{8 f_{\pi}^{2}}-0.3 \frac{\rho}{\rho_{0}} \ldots
$$

where $\rho_{0}=0.16 / \mathrm{fm}^{3}$ is the saturation density of symmetric nuclear matter. This result is model independent and indicates that the mere presence of an ideal gas of hadrons already alters the vacuum structure and leads to a decrease of the condensate, without changing the vacuum properties of the hadrons! Obviously medium-modifications of hadrons and the corresponding non-trivial change of the QCD vacuum has to involve hadronic interactions. They become increasingly important as the medium grows hotter and denser, i.e. as the point of chiral restoration is approached. Thus, the theoretical description in terms of hadrons becomes very complex, involving more and more degrees of freedom. In the vicinity of the restoration transition, in addition, non-perturbative methods are called for which is far from trivial in effective field theories.

In terms of effective fields $\phi_{j}(x)$ representing the pertinent quark currents $J_{\Gamma_{j}}(x)$, hadronic correlation functions in a hot and dense environment are defined as the (retarded) current-current correlation functions

$$
D_{\phi_{j}}(\omega, q)=-i \int d^{4} x \theta\left(x_{0}\right)\left\langle\left\langle\left[\phi_{j}(x), \phi_{j}(0)\right]\right\rangle\right\rangle
$$

Note that, in contrast to the vacuum, these correlators depend on energy $\omega$ and three-momentum $\boldsymbol{q}$ separately since Lorentz invariance is explicitly broken by the presence of matter. Equation (9) can be rewritten in terms of the self energy $\Sigma_{\phi_{i}}$ as

$$
D_{\phi_{j}}(\omega, q)=\left(\omega^{2}-q^{2}-m_{\phi_{j}}^{2}-\Sigma_{\phi_{j}}(\omega, q)\right)^{-1}
$$

where $m_{\phi_{j}}$ denotes the (bare) mass of the field $\phi_{j}$ and all interaction effects are incorporated via $\Sigma_{\phi_{j}}$ which depends on $T$ and $\mu_{B}$. Given the effective Lagrangian, the objective is then to evaluate $\Sigma_{\phi_{j}}$ as realistic as possible. This is usually attempted by employing 'chiral counting rules' for evaluating loop diagrams contributing to $\Sigma_{\phi_{j}}$ in the low-density and low-temperature limit and by adjusting the parameters of the effective Lagrangian to as many data for elementary scattering processes as available. If everything is done consistently, chiral symmetry is properly incorporated and the relation between hadronic medium modifications and vacuum changes can be inferred. 
In the vacuum, the hadronic correlators $D_{\phi_{j}}$ are usually dominated by a few fairly sharp hadronic 'resonances'. These are visible as 'peaks' in the corresponding 'spectral functions'

$$
\rho_{\phi_{j}}(\omega, \boldsymbol{q})=-\frac{1}{\pi} \operatorname{Im} D_{\phi_{j}}(\omega, \boldsymbol{q})
$$

at the hadronic vacuum mass $m_{\phi_{j}}$. Two things will happen in the interacting medium. One the one hand the mass will change to an effective 'pole-mass'

$$
m_{\phi_{j}}^{* 2}=m_{\phi_{j}}^{2}+\operatorname{Re} \Sigma_{\phi_{j}}\left(m_{\phi_{j}}^{* 2}, 0\right) .
$$

In fact, one may have several solutions and the in-medium spectrum shows more than one 'peak'. As we shall see, this is usually the case. On the other hand, the imaginary part $\operatorname{Im} \Sigma_{\phi_{j}}$ acquires additional pieces through the interactions with the medium giving rise to an increased width. If the width becomes too large, the peak structure is washed out and the notion of a 'quasiparticle' is lost. This happens when the 'quasiparticle' energy

$$
\omega_{\phi_{j}}^{2}(\boldsymbol{q})=\boldsymbol{q}^{2}+m_{\phi_{j}}^{2}+\operatorname{Re} \Sigma_{\phi_{j}}\left(\omega_{\phi_{j}}^{2}, q\right)
$$

is no longer large compared to the width

$$
\Gamma_{\phi_{j}}\left(\omega_{\phi_{j}}(q)\right)=-\frac{1}{2 \pi} \operatorname{Im} \Sigma\left(\omega_{\phi_{j}}, q\right) .
$$

This has to be kept in mind when describing in-medium hadrons.

In the following, we will discuss the properties of kaons in the medium and its consequences for the properties of neutron stars. In sect. 2, the elementary kaon-nucleon interaction and the in-medium changes of the kaons as seen in kaonic atoms are described. Chiral effective interactions are constructed which can describe these data and are used to extract the optical potential of kaons at finite density. Section 3 utilizes the results found for the kaon optical potential to apply it to the equation of state for neutron stars. Implications for a first order phase transition to a kaon condensed state are listed. Effects on the onset of kaon condensation by the presence of hyperons in matter are studied. Finally, we summarize in sect. 4 and give an outlook.

\section{Kaons in dense matter}

The properties of kaons and antikaons in the nuclear medium have been the object of numerous investigations since the possibility of the existence of a kaon condensed phase in dense nuclear matter was pointed out by Kaplan and Nelson [5]. If the $K^{-}$meson develops sufficient attraction in dense matter it could be energetically more favorable, after a certain critical density, to neutralize the positive charge with antikaons rather than with electrons. A condensed kaon phase would then start to develop, changing drastically the properties of dense neutron star matter $[6,7,8,9,10,11,12,13,14,15]$. In fact, kaonic atom data, a compilation 
of which is given in [16], favor an attractive $K^{-}$nucleus interaction. On the other hand, the enhancement of the $K^{-}$yield in $\mathrm{Ni}+\mathrm{Ni}$ collisions measured recently by the KaoS collaboration at GSI [17] can be explained by a strong attraction in the medium for the $\mathrm{K}^{-}[18,19,20,21]$. However, the antikaons might feel a repulsive potential at the relatively high temperatures attained in heavy-ion reactions, and an alternative mechanism, based on the production of antikaons via an in-medium enhanced $\pi \Sigma \rightarrow K^{-} p$ reaction, has been suggested [22].

The theoretical investigations that go beyond pure phenomenology [23] have mainly followed two different strategies. One line of approach is that of the mean field models, built within the framework of chiral Lagrangians [21,24,25,26] or based on the relativistic Walecka model which are extended to incorporate strangeness in the form of hyperons or kaons [27] or by using explicitly the quark degrees of freedom [28]. The other type of approach aims at obtaining the in-medium $\bar{K} N$ interaction microscopically by incorporating the medium modifications in a $\bar{K} N$ amplitude using (chiral) effective interactions that reproduces the low energy scattering data and generates the $\Lambda(1405)$ resonance dynamically $[29,30,31,32,33,34,35]$. In this section, we focus on this latter perspective, since it allows one to systematically study the importance of all the different mechanisms that might modify the $\bar{K} N$ interaction in the medium from that in free space.

\section{$2.1 \bar{K} N$ interaction in the medium}

The free $\bar{K} N$ scattering observables $\left(\bar{K}=K^{-}\right.$or $\left.\bar{K}^{0}\right)$ are derived from the scattering amplitude, obtained from the Bethe-Salpeter equation

$$
T=V+V G T \text {, }
$$

which is depicted diagrammatically in Fig. 1. Note that, in the case of $\vec{K}^{-} p$ scattering, this is a coupled-channel equation involving ten intermediate states, namely $K^{-} p, \bar{K}^{0} n, \pi^{0} \Lambda, \pi^{+} \Sigma^{-}, \pi^{0} \Sigma^{0}, \pi^{-} \Sigma^{+}, \eta \Lambda, \eta \Sigma^{0}, K^{+} \Xi^{-}$and $K^{0} \Xi^{0}$. The loop operator $G$ stands for the diagonal intermediate meson-baryon $(M B)$ propagator and $V$ is a suitable $M B \rightarrow M^{\prime} B^{\prime}$ transition potential.

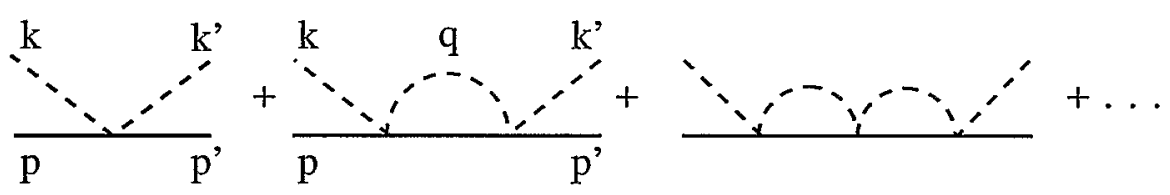

Fig. 1. Diagrammatic representation of the Bethe-Salpeter equation for $\bar{K} N$ scattering.

A connection with the chiral Lagrangian was established in [3], where the properties of of the $S=-1$ meson-baryon sector were studied in a potential 
model [3], such that, in Born approximation, it had the same S-wave scattering length as the chiral Lagrangian, including both the lowest order and the momentum dependent $p^{2}$ terms. No $\eta \Lambda, \eta \Sigma$ or $K \Xi$ channels were considered, on the basis that they were not opened at the $K^{-} p$ threshold. By fitting five parameters, corresponding to, so far, unknown parameters of the second order chiral Lagrangian plus the range parameters of the potential, the $A(1405)$ resonance was generated as a quasi-bound meson-baryon state and the $K^{-} p \rightarrow M B$ cross sections, as well as the available branching rations at threshold, were well reproduced. Note that being close to a resonance forces one to resort to nonperturbative approaches, such as summing the infinite Bethe-Salpeter series as represented diagrammatically in Fig. 1. A recent work [36], which shares many points with [3], showed that all the strangeness $S=-1$ meson-baryon scattering observables near threshold could be reproduced using only the lowest order Lagrangian in the Bethe-Salpeter equation and one parameter, the cut-off $q_{\max }$ that regularizes the loop function $G$. All 10 meson-baryon states that can be generated from the octet of pseudoscalar mesons and the octet of ground-state baryons were included, the additional $\eta \Lambda$ state being a quite relevant one. The success of this method in reproducing the scattering observables is analogous to that obtained in the meson-meson sector [37]. An explanation was found in [38] by applying the Inverse Amplitude Method in coupled channels to the same problem, with the lowest and next-to-lowest order meson-meson Lagrangian. It was shown that the selection of an appropriate cut off for a particular $I, J$ channel could minimize the contribution of the next-to-lowest order Lagrangian, reducing the relevant higher order terms to those iterated by the Bethe-Salpeter equation with the lowest order Lagrangian, which is the simplified method followed in [36,37] (see also the discussion in the review [39]).

The most obvious medium effect is that induced by Pauli-blocking on the nucleons in the intermediate states. This makes the $\bar{K} N$ interaction density dependent and modifies, in turn, the $K^{-}$properties from those in free space. These effects were already included a long time ago in the context of Bruecknertype many body theory, using a separable $\bar{K} N$ interaction [29]. Some more recent works $[31,32,33,34,35]$ take the $\bar{K} N$ interaction from the chiral Lagrangian in Swave. However, as seen in the recent work by Tolos et al. [40] which uses the Bonn $\bar{K} N$ potential model [41], the incorporation of higher angular momenta has non-negligible effects on the properties of the antikaon at high momentum, which is of relevance for the analysis of in-medium effects for the $\mathrm{K}^{-}$in heavy-ion collisions.

In the actual calculations, the effect of Pauli blocking is incorporated by replacing the free nucleon propagator in the loop function $G$ for intermediate $\bar{K} N$ states by an in-medium one of the type

$$
A\left(\sqrt{s}-q^{0},-\boldsymbol{q}, \rho\right)=\frac{1-n\left(\boldsymbol{q}_{\mathrm{lab}}\right)}{\sqrt{s}-q^{0}-E(-\boldsymbol{q})+i \epsilon}+\frac{n\left(\boldsymbol{q}_{\mathrm{lab}}\right)}{\sqrt{s}-q^{0}-E(-\boldsymbol{q})-i \epsilon},
$$

where $n\left(\boldsymbol{q}_{\mathrm{lab}}\right)$ is the occupation probability of a nucleon of momentum $\boldsymbol{q}_{\mathrm{lab}}$ in the lab frame. 
The most spectacular consequence of the Pauli principle is that the blocking of intermediate states shifts the resonance to higher energy. This changes the $\bar{K} N$ interaction at threshold from being repulsive in free space to being attractive in the medium. Therefore, antikaons develop an attractive optical potential which, incorporated again in the $\bar{K} N$ states of the in-medium scattering equation, may compensate the upward shifting of these intermediate states induced by Pauli blocking. This feedback has been recently confirmed by the calculation of Lutz [34], where the dressing of the antikaon is incorporated in the intermediate loops in a self-consistent manner. The $\Lambda(1405)$ resonance remains then unchanged, in qualitative agreement with what was noted in [30] using a constant mean field potential for the $\vec{K}$.

Since the dressing of the antikaon turns out to be so relevant, one might wonder about dressing the other mesons or baryons that play a role in the $\bar{K} N$ system. This has been explored in the recent work [35], where a self-consistent antikaon self-energy is obtained including the dressing of the pions in the $\pi \Lambda$, $\pi \Sigma$ intermediate states, which are the ones that couple strongly to $\vec{K} N$.

Incorporating the medium modified mesons in the calculation is technically achieved by replacing the free meson propagator in the loop function $G$ by

$$
D\left(q^{0}, \boldsymbol{q}, \rho\right)=\frac{1}{\left(q^{0}\right)^{2}-\boldsymbol{q}^{2}-m^{2}-\Pi\left(q^{0}, \boldsymbol{q}, \rho\right)}=\int_{0}^{\infty} d \omega 2 \omega \frac{S(\omega, \boldsymbol{q}, \rho)}{\left(q^{0}\right)^{2}-\omega^{2}+i \epsilon},
$$

where $\Pi\left(q^{0}, q, \rho\right)$ is the meson self-energy. The Lehman representation shown in the second equality of (17) introduces the spectral density, $S(\omega, \boldsymbol{q}, \rho)=$ $-\operatorname{Im} D(\omega, \boldsymbol{q}, \rho) / \pi$, which in the case of free mesons reduces to $\delta(\omega-\omega(\boldsymbol{q})) / 2 \omega(\boldsymbol{q})$. With these modifications the loop integral becomes

$$
\begin{aligned}
G\left(P^{0}, \boldsymbol{P}, \rho\right) & =\int_{|\boldsymbol{q}|<q_{\max }} \frac{d^{3} q}{(2 \pi)^{3}} \frac{M}{E(-\boldsymbol{q})} \int_{0}^{\infty} d \omega S(\omega, \boldsymbol{q}, \rho) \\
& \times\left\{\frac{1-n\left(\boldsymbol{q}_{\mathrm{lab}}\right)}{\sqrt{s}-\omega-E(-\boldsymbol{q})+i \epsilon}+\frac{n\left(\boldsymbol{q}_{\mathrm{lab}}\right)}{\sqrt{s}+\omega-E(-\boldsymbol{q})-i \epsilon}\right\},
\end{aligned}
$$

where $\left(P^{0}, \boldsymbol{P}\right)$ is the total four-momentum in the lab frame and $s=\left(P^{0}\right)^{2}-\boldsymbol{P}^{2}$. Note that the loop function $G$ does-not contain the $V$ and $T$ amplitudes. This simplification is possible in the chiral approach of [36], where it was shown that the the amplitudes factorize on-shell out of the loop, since the off shell part could be absorbed into a renormalization of the coupling constant $f_{\pi}$. These arguments can not be applied in potential models and a coupled system of integral equations must be solved. A reasonable simplification is obtained if the dressing of the antikaon in the loop function $G$ is taken into account via an energy-independent self-energy, $\Pi\left(q^{0}=\varepsilon_{q p}(\boldsymbol{q}), \boldsymbol{q}, \rho\right)$, evaluated at the quasiparticle energy, $\varepsilon_{q p}(\boldsymbol{q})$, which is the solution of the in-medium dispersion relation

$$
\varepsilon_{q p}^{2}(\boldsymbol{q})=\boldsymbol{q}^{2}+m_{K}^{2}+\Pi\left(q^{0}=\varepsilon_{q p}(\boldsymbol{q}), \boldsymbol{q}, \rho\right) .
$$

This is the prescription followed in $[22,40]$ and amounts to approximate the actual antikaon spectral function by a symmetric pseudo-Lorentzian peak at the 
quasiparticle energy given by (19). With this assumption, the loop function $G$ looks like the free one, but replacing the free antikaon energy $\sqrt{q^{2}+m_{K}^{2}}$ by the complex quasiparticle one, $\varepsilon_{q p}(\boldsymbol{q})$.

One might also include the dressing of the baryons by assuming that the single particle energy $E(-q)$ in (16) to contain a mean-field potential of the type $U_{0} \times \rho / \rho_{0}$, as done in $[35,40]$. For the nucleon, a reasonable depth value is $U_{0}^{N}=-70 \mathrm{MeV}$, as suggested by numerous calculations of the nucleon potential in nuclear matter. For the $\Lambda$ hyperon, one can take $U_{0}^{A}=-30 \mathrm{MeV}$, as implied by extrapolating the experimental $\Lambda$ single particle energies in $\Lambda$ hypernuclei to bulk matter [42]. For the $\Sigma$ hyperon, there is no conclusive information on the potential. Early phenomenological analyzes [43] and calculations [44] found the $\Sigma$ atomic data to be compatible with $U_{0}^{\Sigma} \sim-30 \mathrm{MeV}$, but more recent analysis indicate a repulsive potential in the nuclear interior [45]. As shown in [35], changing the $\Sigma$ depth from -30 to $+30 \mathrm{MeV}$ does not change the results for the properties of the $\mathrm{K}^{-}$in the medium considerably.

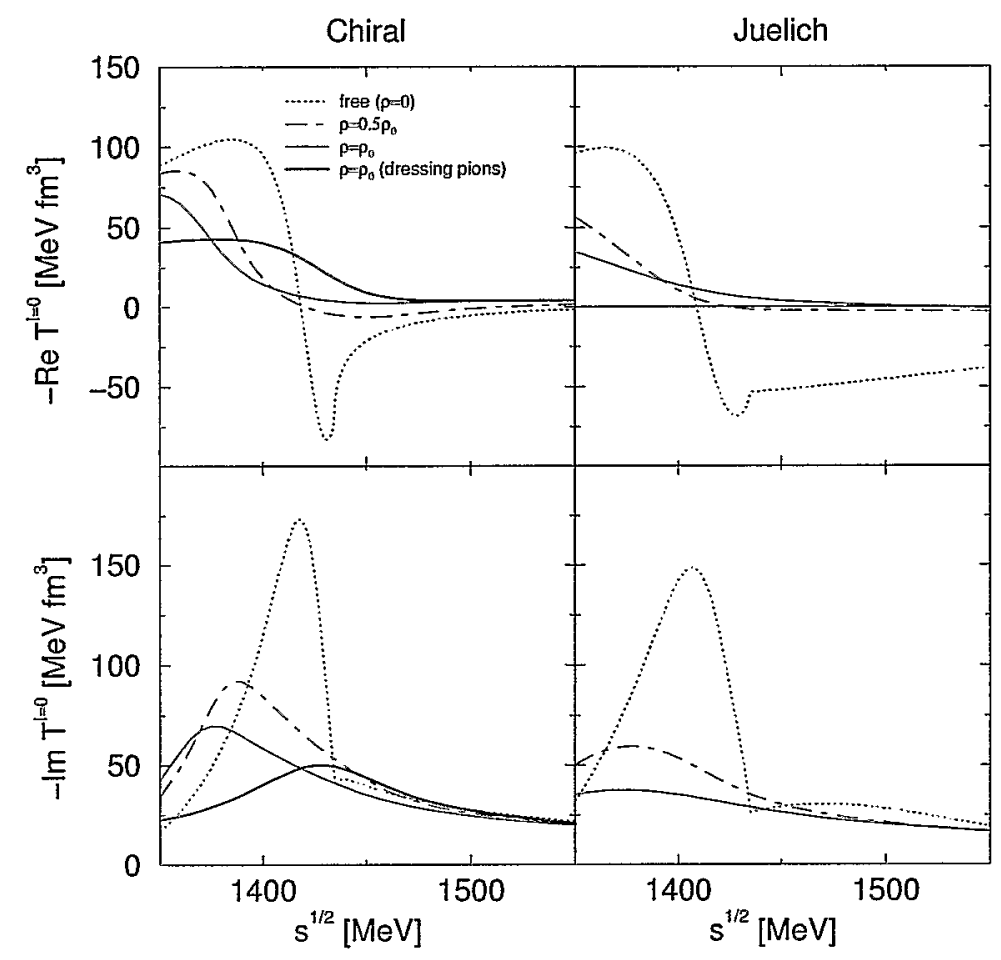

Fig. 2. Real and imaginary parts of the $I=0 \bar{K} N$ scattering amplitude as functions of $\sqrt{s}$ for $\left|p_{K}+p_{N}\right|=0$ and several densities (from [35] and [40]). 
Using the dressed meson-baryon loop in the coupled-channel Bethe-Salpeter equation, one obtains the in-medium $\bar{K} N$ amplitude $T_{\text {eff }}\left(P^{0}, \boldsymbol{P}, \rho\right)$. In Fig. 2 we compare the free amplitude ( $\rho=0$, dotted line) in the $I=0, L=0$ channel with that obtained at nuclear densities $\rho=\rho_{0}=0.17 \mathrm{fm}^{-1}$ (solid line) and $\rho=0.5 \rho_{0}$ (dot-dashed line). Two different models are shown, that of [35], based on the lowest order meson-baryon chiral Lagrangian, and that of [40], based on the potential model of the Bonn group [41]. In spite of the appreciable differences seen in the free scattering amplitudes, the medium modified ones show the same qualitative trends. Note also how the real part of the amplitude (upper panels) at the $K^{-} p$ threshold $(\sqrt{s}=1433 \mathrm{MeV})$ is repulsive in free space and attractive in the medium. The thick solid line on the left panels show the effect on the $K^{-} p$ amplitude by dressing the pions in the intermediate states [35].

Most of the available models study the in-medium $\bar{K} N$ amplitude in S-wave. However, if one aims at extracting the properties of antikaons through the analysis of heavy-ion collisions, one must keep in mind that they are created at a finite momentum of around $250-500 \mathrm{MeV} / \mathrm{c}$, hence the effect of higher partial waves might be relevant. The meson-exchange $\bar{K} N$ potential of the Jülich group [41] is given in partial waves and the main results from a recent study [40] on the effect of the angular momentum states higher than the commonly considered $L=0$ one will be summarized in the next subsection. From the chiral perspective, the P-wave amplitudes from the next-to-leading order $\bar{K} N$ chiral Lagrangian have been identified and, recently, the parameters have been fitted to reproduce a large amount of low energy data [46]. However, a nuclear medium application of this model is not available yet.

\subsection{In medium $\bar{K}$ properties}

The $\bar{K}$ self-energy is obtained by summing the in-medium $\bar{K} N$ interaction, $T_{\text {eff }}\left(P^{0}, P, \rho\right)$, over the nucleons in the Fermi sea

$$
\Pi_{\bar{K}}\left(q^{0}, \boldsymbol{q}, \rho\right)=2 \sum_{N=n, p} \int \frac{d^{3} p}{(2 \pi)^{3}} n(\boldsymbol{p}) T_{\mathrm{eff}}\left(q^{0}+E(\boldsymbol{p}), \boldsymbol{q}+\boldsymbol{p}, \rho\right) .
$$

Note that a self-consistent approach is required since one calculates the $\vec{K}$ selfenergy from the effective interaction $T_{\text {eff }}$ which uses $\bar{K}$ propagators which themselves include the self-energy being calculated.

A P-wave contribution to the $\bar{K}$ self-energy coming from the coupling of the $\bar{K}$ meson to hyperon-hole excitations can be easily included (if it is not already contained in $T_{\text {eff }}$ ) and the expression can be found in [35]. In that work the pions are also dressed through a pion self-energy that contains one- and twonucleon absorption and is conveniently modified to include the effect of nuclear short-range correlations (see [47] for details). The resulting pion spectral density in nuclear matter at density $\rho=\rho_{0}$ is shown in Fig. 3 as a function of the pion energy for several momenta. The strength is distributed over a wide range of energies and, as the pion momentum increases, the position of the peak is increasingly lowered from the corresponding one in free space as a consequence 
of the attractive pion-nuclear potential. Note that, to the left of the peaks, there appears the typical structure of the $1 p 1 h$ excitations which give rise to $1 p 1 h \Lambda$ and $1 p 1 h \Sigma$ components in the effective $\bar{K} N$ interaction. Although not visible in the linear scale used in Fig. 3, there is some additional strength at energies around $300 \mathrm{MeV}$ associated to the excitation of the $\Delta$ resonance.

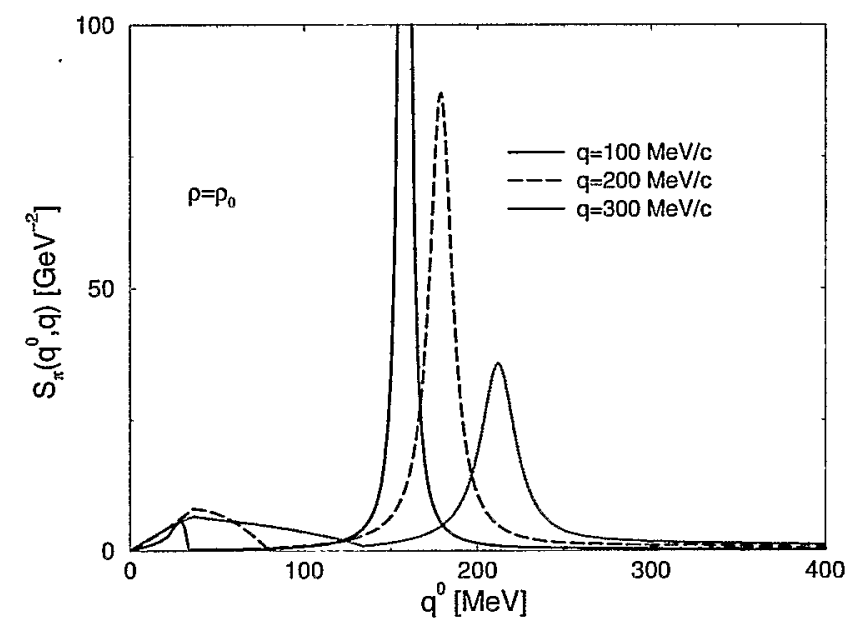

Fig. 3. Pion spectral density at $\rho=\rho_{0}$ for several momenta (from [35]).

The spectral function of a $K^{-}$meson of zero momentum obtained with the chiral model of [35] is shown in Fig. 4 for various densities: $\rho_{0}, \rho_{0} / 2$ and $\rho_{0} / 4$. The results in the upper panel include only Pauli blocking effects, i.e. the nucleons propagate as in (16) but the mesons behave as in free space. At $\rho_{0} / 4$ one clearly sees two excitation modes. The left one corresponds to the $K^{-}$pole branch, appearing at an energy smaller than the kaon mass, $m_{K}$, due to the attractive medium effects. The peak on the right corresponds to the $\Lambda(1405)$ hole excitation mode, located above $m_{K}$ because of the shifting of the $\Lambda(1405)$ resonance to energies above the $K^{-} p$ threshold. As density increases, the $K^{-}$ feels an enhanced attraction while the $A(1405)$-hole peak moves to higher energies and loses strength, a reflection of the tendency of the $A(1405)$ to dissolve in the dense nuclear medium. These features were already observed in $[31,33]$. The (self-consistent) incorporation of the $\bar{K}$ propagator in the Bethe-Salpeter equation softens the effective interaction, $T_{\text {eff }}$, which becomes more spread out in energies (solid and dot-dashed lines in Fig. 2). The resulting $K^{-}$spectral function (middle panel in Fig. 4) shows the displacement of the resonance to lower energies because, as already noted, the attraction felt by the $\bar{K}$ meson lowers the threshold for the $\bar{K} N$ states that had been increased by the Pauli blocking on the nucleons. This has a compensating effect and the resonance moves backwards, slightly below its free space value. The $K^{-}$pole peak appears at 


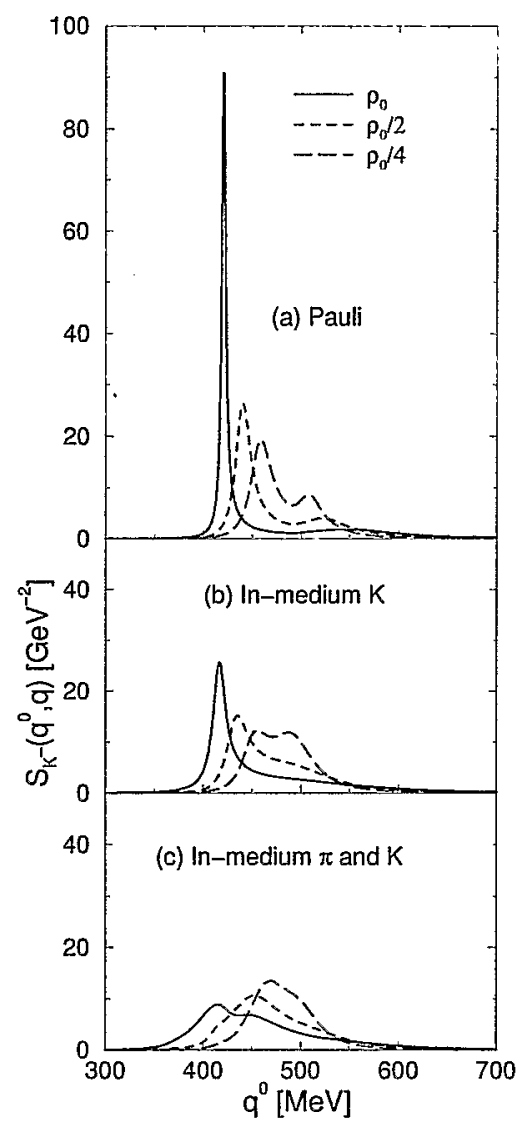

Fig.4. $K^{-}$spectral density for zero momentum from the chiral model of ref. [35]

similar or slightly smaller energies, but its width is larger, due to the strength of the intermediate $\bar{K} N$ states being distributed over a wider region of energies. Therefore the $K^{-}$pole and the $\Lambda(1405)$-hole branches merge into one another and can hardly be distinguished. Finally, when the pion is dressed according to the spectral function shown in Fig. 3 the effective interaction $T_{\text {eff }}$ becomes even smoother (thick solid lines in Fig. 2). The resulting $K^{-}$spectral function is displayed in the bottom panel in Fig. 4. Even at very small densities one can no longer distinguish the $\Lambda(1405)$-hole peak from the $K^{-}$pole one. As density increases, the attraction felt by the $K^{-}$is more moderate and the $K^{-}$pole peak appears at higher energies than in the other, two approaches. However, more strength is found at very low energies, especially at $\rho_{0}$, due to the $1 p 1 h 2 p 2 h$ components of the pionic strength, which couple the $\bar{K} N$ state to the $1 p 1 h \Sigma$ and $2 p 2 h \Sigma$ ones. It is precisely the opening of the $\pi \Sigma$ channel, on top of the already opened $1 p 1 h \Sigma$ and $2 p 2 h \Sigma$ ones, which causes a cusp structure to appear slightly above $400 \mathrm{MeV}$. 


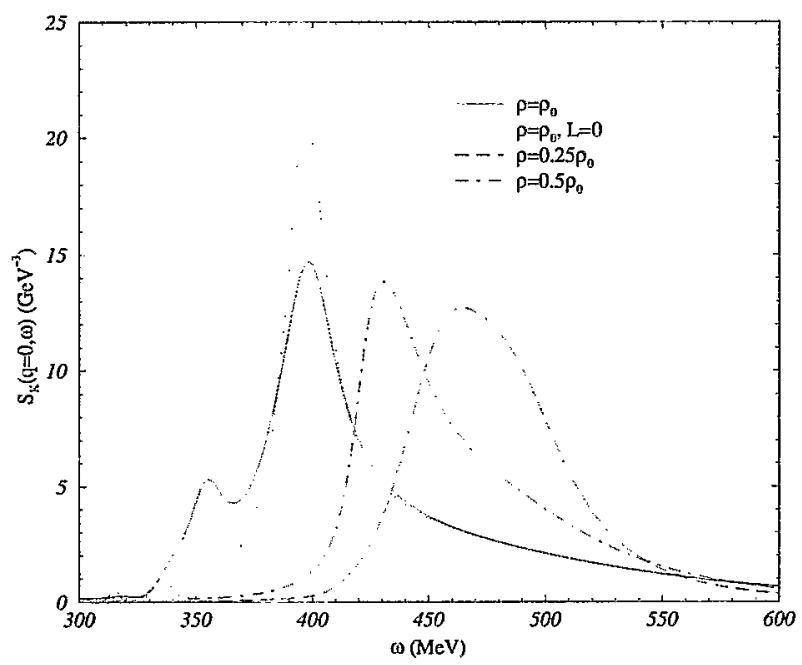

Fig. 5. $K^{-}$spectral density for zero momentum using the Bonn $\bar{K} N$ potential (from [40]).

The calculation of [40] using the Bonn $\vec{K} N$ potential obtains similar results, which are shown in Fig. 5 for the same three densities. We notice some structure of the spectral function to the left of the quasiparticle peak at energies of the $\bar{K}$ around $320-360 \mathrm{MeV}$. This is the in-medium reflection of a singularity in the $L=1, I=1$ free space amplitude around the mass of the $\Sigma$ baryon induced by the $\Sigma$-pole diagram present in the Bonn $\bar{K} N$ potential [41]. This peak is therefore indicating the physical excitation of $\Sigma h$ states with antikaon quantum numbers. The dotted line shows the spectral density at $\rho=\rho_{0}$ but keeping only the $L=0$ component of the $\bar{K} N$ interaction. In agreement with the behavior of the complex antikaon potential at zero momentum shown below, we observe that the location of the quasiparticle peak (driven by the real part) only moves a few $\mathrm{MeV}$, while the width (driven by the imaginary part) gets reduced by about $30 \%$ when including higher partial waves.

One may define a non-relativistic antikaon single-particle potential from the self-energy at the quasiparticle energy via the relation

$$
U_{K}(q)=\frac{\Pi_{\bar{K}}\left(\varepsilon_{q p}(q), q, \rho\right)}{2 m_{K}} .
$$

The real and imaginary parts of the antikaon potential at $\rho=\rho_{0}$, obtained from the chiral model of ref. [35], are shown in Fig. 6 as function of the antikaon momentum for two approximations, one in which only the antikaon self-energy is considered in the intermediate loops (thin solid lines) and another in which the pions are also dressed (thick solide line). Note that the antikaon potential obtained when the pions are also dressed has much less structure. This is due to the smoother in-medium amplitude, but also to the different quasiparticle 


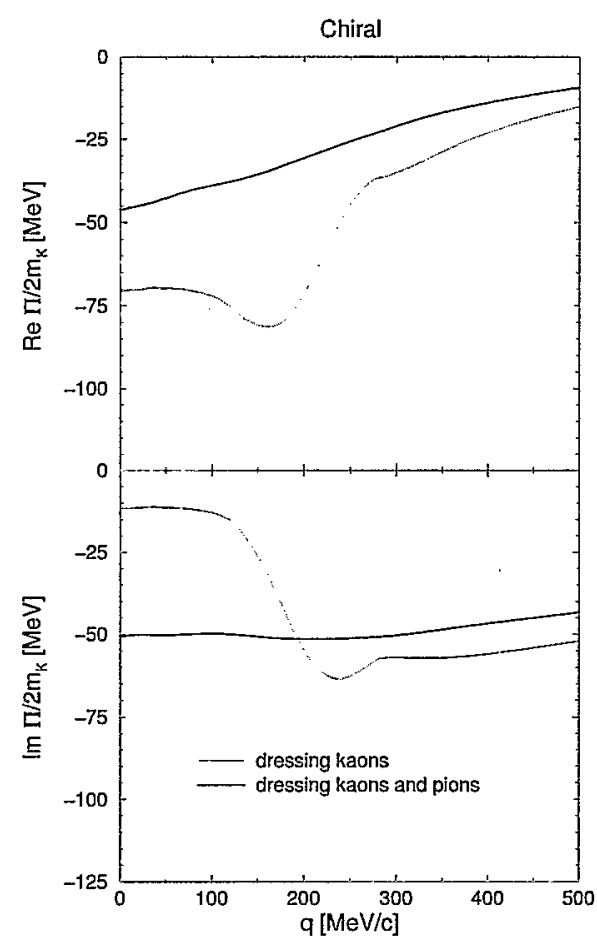

Fig. 6. Real and imaginary parts of the $\bar{K}$ optical potential at $\rho=\rho_{0}$ as functions of the antikaon momentum, as obtained from the chiral model of [35].

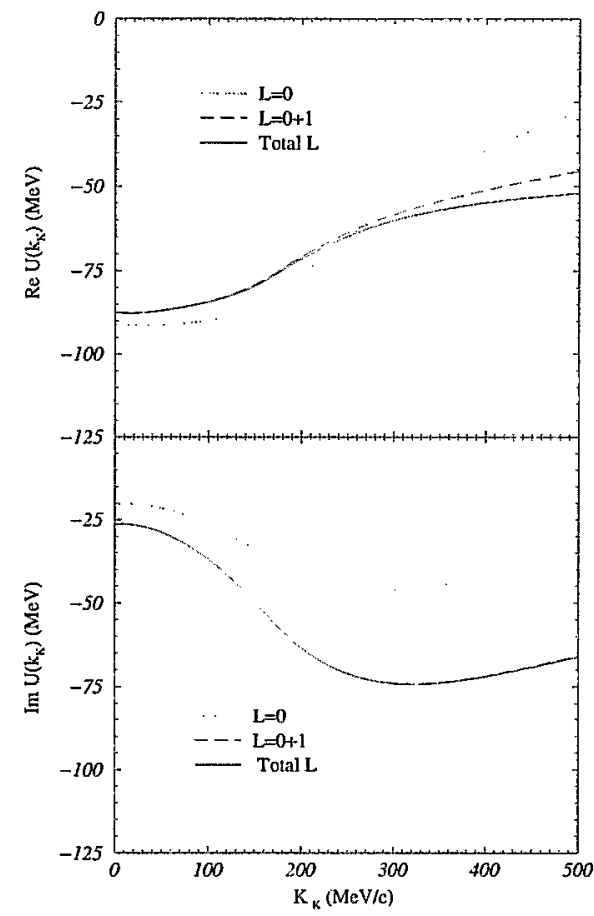

Fig. 7. The same as Fig. 6, but obtained using the Bonn $\bar{K} N$ potential (taken from [40]).

energy at which the antikaon self-energy is evaluated. This quasiparticle energy is more attractive when only antikaons are dressed and, hence, the amplitude is explored at lower energy regions, closer to the position of the in-medium $A(1405)$ resonance. Dressing the intermediate pions gives an antikaon potential depth at zero momentum of $-45 \mathrm{MeV}$. This is about half the attraction of that obtained with other recent models and approximation schemes [21,26,27,28,32,33], which give rise to potential depths at the center of the nucleus between -140 and -75 $\mathrm{MeV}$. Results obtained by the model of [40] using the Bonn $\bar{K} N$ potential [41], where only kaons are dressed, are shown in Fig. 7. In this figure one can also see the effect of including the higher partial waves of the Bonn $\bar{K} N$ interaction. We observe that the antikaon nuclear potential at zero momentum receives some contribution of partial waves higher than $L=0$, due to the fact that the $\bar{K}$ meson interacts with nucleons that occupy states up to the Fermi momentum, giving rise to finite $\bar{K} N$ relative momenta of up to around $90 \mathrm{MeV} / \mathrm{c}$. Clearly, the effect of the higher partial waves increases with increasing $\bar{K}$ momentum, flattening out the real part of the potential and producing more structure to the imaginary part. At an antikaon momentum of around $500 \mathrm{MeV} / \mathrm{c}$, the inclusion 
of the higher partial waves practically doubles the size of the antikaon potential with respect to the $\mathrm{S}$-wave value.

\subsection{Kaonic atoms}

Since the $K^{-}$in kaonic atoms are bound with small (atomic) energies, their study requires the knowledge of the antikaon self-energy at $\left(q^{0}, q\right)=\left(m_{K}, 0\right)$. The real and imaginary parts of the isospin averaged in-medium scattering length, defined as

$$
a_{\mathrm{eff}}(\rho)=-\frac{1}{4 \pi} \frac{M}{m_{K}+M} \frac{\Pi_{\bar{K}}\left(m_{K}, q=0, \rho\right)}{\rho},
$$

obtained from the chiral model of [35], are shown in Fig. 8 as function of the nuclear density $\rho$. The change of the real part of the effective scattering length Re $a_{\text {eff }}$ from negative to positive values indicates the transition from a repulsive interaction in free space to an attractive one in the medium. As shown by the dotted line, this transition happens at a density of about $\rho \sim 0.1 \rho_{0}$ when only Pauli effects are considered, in agreement with what was found in [31,32]. However, this transition occurs at even lower densities $\left(\rho \sim 0.04 \rho_{0}\right)$ when one considers the self-energy of the mesons in the description, whether one dresses only the $\bar{K}$ meson (dashed line) or both the $\bar{K}$ and $\pi$ mesons (solid line). The deviations from the approach including only Pauli blocking or those dressing the mesons are quite appreciable over a wide range of densities. The thin solid lines show the results obtained with a repulsive $\Sigma$ potential depth of $+30 \mathrm{MeV}$. The deviations from the thick solid line, obtained for an attractive potential depth of $-30 \mathrm{MeV}$, are smaller than $10 \%$ and only show up at higher densities.

The implications of the density dependent scattering length displayed in Fig. 8 on kaonic atoms have been recently analyzed [48] in the framework of a local density approximation, which amounts to replace the nuclear matter density $\rho$ by the density profile $\rho(r)$ of the particular nucleus. The results displayed in Fig. 9 show that both the energy shifts and widths of kaonic atom states agree well with the bulk of experimental data [49].

Reproducing kaonic atom data with this moderately attractive antikaon nucleus potential of $-45 \mathrm{MeV}$ is in contrast with the depth of around $-200 \mathrm{MeV}$ obtained from a best fit to $K^{-}$atomic data with a phenomenological potential that includes an additional non-linear density dependent term [16]. A hybrid model, combining a relativistic mean field approach in the nuclear interior and a phenomenological density dependent potential at the surface that is fitted to $K^{-}$atomic data, also favors a strongly attractive $K^{-}$potential of depth -180 $\mathrm{MeV}$ [50]. On the other hand, the early Brueckner-type calculations of [29] also obtained a shallow $\mathrm{K}^{-}$-nucleus potential, of the order of $-40 \mathrm{MeV}$ at the center of ${ }^{12} \mathrm{C}$, and predicted reasonably well the $K^{-}$atomic data available at that time. Acceptable fits to kaonic atom data have also been obtained using charge densities and a phenomenological $T_{\text {eff }} \rho$ type potential with a depth of the order of $-50 \mathrm{MeV}$ in the nuclear interior [51], which goes down to $-80 \mathrm{MeV}$, when matter densities are used instead [16]. 


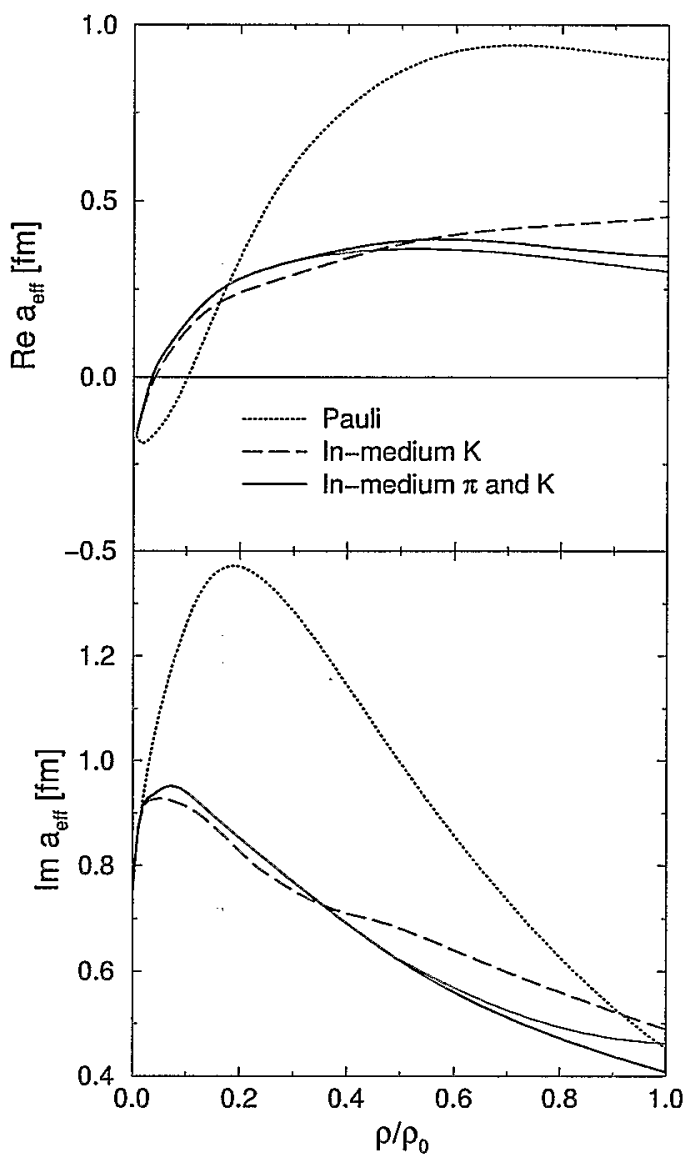

Fig. 8. $K^{-} N$ scattering length as a function of density (from [35]).

A clarifying quantitative comparison of kaonic atom results obtained with various $K^{-}$-nucleus potentials can be found in [52]. There, the reasonable reproduction of data obtained with the chiral antikaon-nucleus potential of [35], shown in Fig. 9, is quantified with a $\chi^{2} /$ d.o.f. $=3.8$. This potential is then modified by an additional phenomenological piece, linear in density, which is fitted to the known data and is able to bring the agreement to the level of $\chi^{2} /$ d.o.f. $=1.6$. This results into a final potential which is slightly more attractive $\left(-50 \mathrm{MeV}\right.$ at $\left.\rho_{0}\right)$ and has a reduced imaginary part by about a factor 2 . The work [52] reemphasizes that kaonic atoms only explore the antikaon potential at the surface of the nucleus. Therefore, although all models predict attraction for the $K^{-}$-nucleus potential, the precise value of its depth at the center of the nucleus, which has important implications for the occurrence of kaon condensation, is still not known. It is then necessary to gather more data that could help in disentangling the properties of the $\bar{K}$ in the medium. Apart from the 


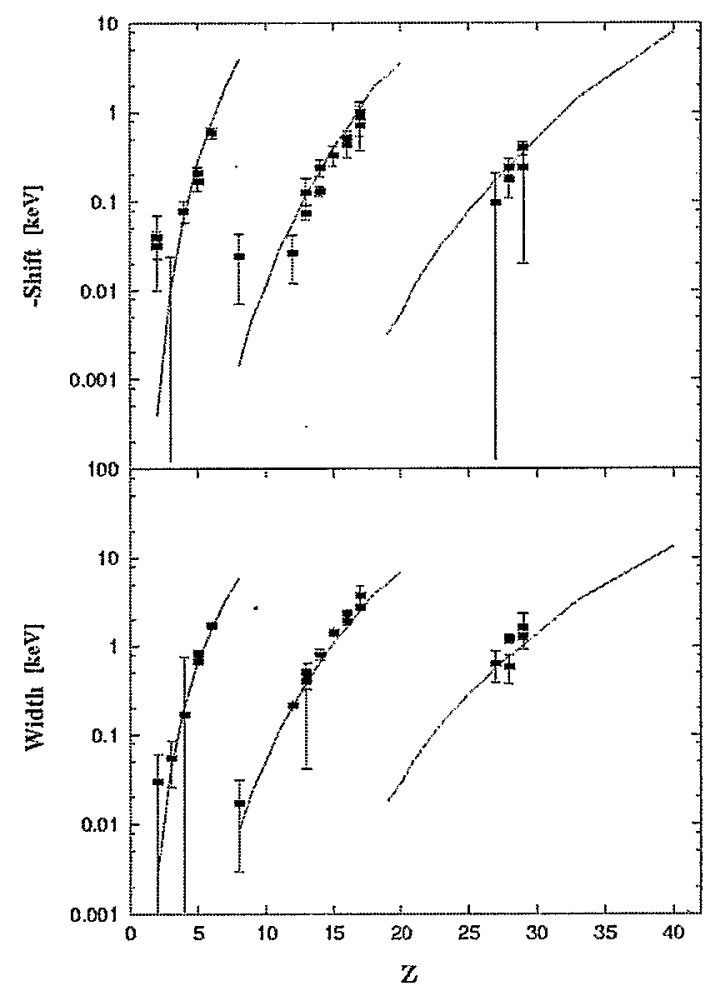

Fig. 9. Energy shifts and widths of kaonic atom states (from [48]). The experimental data are taken from the compilation given in [49].

valuable information that can be extracted from the production of $K^{-}$in heavyion collisions, one could also measure deeply bound kaonic states, which have been predicted to be narrow $[48,52,53]$ and could be measured in $\left(K^{-}, \gamma\right)[48]$ or $\left(K^{-}, p\right)$ reactions $[54,55]$.

\section{Kaons in neutron stars}

Kaon condensation has first thought to be irrelevant for neutron stars as their mass has to be lowered so drastically to appear in beta-stable neutron star matter [56]. Nevertheless, as demonstrated in the last sections, the in-medium effects for the kaons, especially for the $\mathrm{K}^{-}$can be quite pronounced which reopened the issue of kaon condensation for neutron stars [5]. The topic has been extensively discussed in the literature over the last years (see e.g. $[6,7,8,9,10,11,12,13,14])$. In all these approaches, the antikaon-nucleon interaction has been parameterized 
in effective field theoretical models which were guided by the investigations of the last sections. So far, a consistent coupled channel calculation incorporating a realistic nucleon-nucleon interactions as well as kaon-nucleon interactions has not been performed due to the complexity of the problem. We will outline in this section, how one can parameterize the antikaon-nucleon interaction in a simple field theoretical model and apply it to the equation of state (EoS) of beta-stable matter and neutron stars.

\subsection{Effective model of kaon-nucleon interactions}

In neutron star matter, only baryon number and charge are conserved. Hence, kaons or antikaons, as well as hyperons, can appear inside neutron stars by strangeness changing processes. The onset of the appearance of the negatively charged $\mathrm{K}^{-}$is given by the equality of the effective antikaon chemical potential (or effective antikaon energy) in matter with the electrochemical potential

$$
\omega_{K}=\mu_{K^{-}}=\mu_{e} \text {. }
$$

Then processes like

$$
e^{-} \rightarrow K^{-}+\nu_{e} \quad n \rightarrow p+K^{-}
$$

are energetically allowed. The $\mathrm{K}^{-}$is replacing electrons from the Fermi surface or equivalently transforming neutrons to protons. The $\mathrm{K}^{-}$as a boson will form a condensate with zero momenta as the s-wave interaction with nucleons is attractive. The presence of the zero momentum $\mathrm{K}^{-}$'s, compared to the high momenta electrons, will lower the overall energy of the system. Also, the increase in the proton fraction will lower the isospin asymmetry of the matter. As the nuclear asymmetry term is strongly repulsive, $\mathrm{K}^{-}$can again lower the energy of the system substantially.

Guided by the discussion of the previous sections, we write down now an effective Lagrangian which models the kaon-nucleon interaction:

$$
\mathcal{L}_{K}=\mathcal{D}_{\mu}^{*} K^{*} \mathcal{D}^{\mu} K-m_{K}^{*} K^{*} K
$$

where the vector fields are coupled minimally

$$
\mathcal{D}_{\mu}=\partial_{\mu}+i g_{\omega K} V_{\mu}+i g_{\rho K} \boldsymbol{\tau}_{K} \boldsymbol{R}_{\mu}
$$

and the effective mass of the kaon is defined as a linear shift of the mass term by the scalar field

$$
m_{K}^{*}=m_{K}-g_{\sigma K} \sigma
$$

The form of the interaction as mediated by a scalar $(\sigma)$ and vector $\left(V_{\mu}, \boldsymbol{R}_{\mu}\right)$ meson fields is in close analogy to the relativistic mean-field model which will be used for the baryon-baryon interactions. We will focus now on the $\mathrm{K}^{-}$. The combined equations of motion for the meson fields including nucleons

$$
m_{\sigma}^{2} \sigma+b m_{N}\left(g_{\sigma N} \sigma\right)^{2}+c\left(g_{\sigma N} \sigma\right)^{3}=g_{\sigma N} \rho_{s}+g_{\sigma K} \rho_{K}
$$




$$
\begin{array}{ll}
m_{\omega}^{2} V_{0} & =g_{\omega N}\left(\rho_{p}+\rho_{n}\right)-g_{\omega K} \rho_{K} \\
m_{\rho}^{2} R_{0,0} & =g_{\rho N}\left(\rho_{p}-\rho_{n}\right)-g_{\rho K} \rho_{K}
\end{array}
$$

has a certain simple structure. The scalar densities for nucleons and $\mathrm{K}^{-}$act as a source term for the scalar field. The vector densities are conserved and build source terms for the corresponding vector fields. The different signs for the source terms of the isospin dependent $R_{0}$ field reflect the isospin of the hadron. Note, that the kaon scalar and vector density are equal in our approach as the kaon has spin zero, contrary to the nucleon. The dispersion relation for the $\mathrm{K}^{-}$at zero momentum is given by

$$
\omega_{K}=m_{K}-g_{\sigma K} \sigma-g_{\omega K} V_{0}-g_{\rho K} R_{0,0} .
$$

The solution of the equations of motion provides then an EoS of the form

$$
\begin{aligned}
& \epsilon=\epsilon_{N}+\epsilon_{K}+\epsilon_{e, \mu} \\
& p=p_{N}+p_{e, \mu} .
\end{aligned}
$$

Note, that the direct contribution of the kaons to the pressure vanishes, as it involves a Bose condensate. The energy contribution of the $\mathrm{K}^{-}$reads

$$
\epsilon_{K}=m_{K}^{*} \rho_{K}
$$

Now there are two parameters for the $\mathrm{K}^{-}$, which have to be fixed. For the vector coupling constants, we use simple quark counting rules and set

$$
g_{\omega K}=\frac{1}{3} g_{\omega N} \quad \text { and } \quad g_{\rho K}=g_{\rho N}
$$

The scalar coupling constant is fixed to the optical potential of the $\mathrm{K}^{-}$at $\rho_{0}$ :

$$
U_{K}\left(\rho_{0}\right)=-g_{\sigma K} \sigma\left(\rho_{0}\right)-g_{\omega K} V_{0}\left(\rho_{0}\right)
$$

and will be varied between -140 and $-80 \mathrm{MeV}$ according to the results of the coupled channel calculations of the previous section.

\subsection{Phase transition to kaon condensation}

The phase transition to kaon condensed matter can be in principle of any order. If the phase transition is of first order, then Gibbs' general condition for two phases have to be applied. As there are two conserved charges for cold neutron star matter, baryon number and charge, the Gibbs conditions read

$$
p^{\mathrm{I}}=p^{\mathrm{II}}, \quad \mu_{B}^{\mathrm{I}}=\mu_{B}^{\mathrm{II}}, \quad \mu_{e}^{\mathrm{I}}=\mu_{e}^{\mathrm{II}}
$$

in kinetic and chemical equilibrium. Note, that the standard Maxwell construction can not be used, as it ensures to conserve only one chemical potential.

For a sufficiently large attraction for the $\mathrm{K}^{-}, U_{K}\left(\rho_{0}\right)>-80 \mathrm{MeV}$, we find that the phase transition is indeed of first order. Figure 10 shows the EoS of 


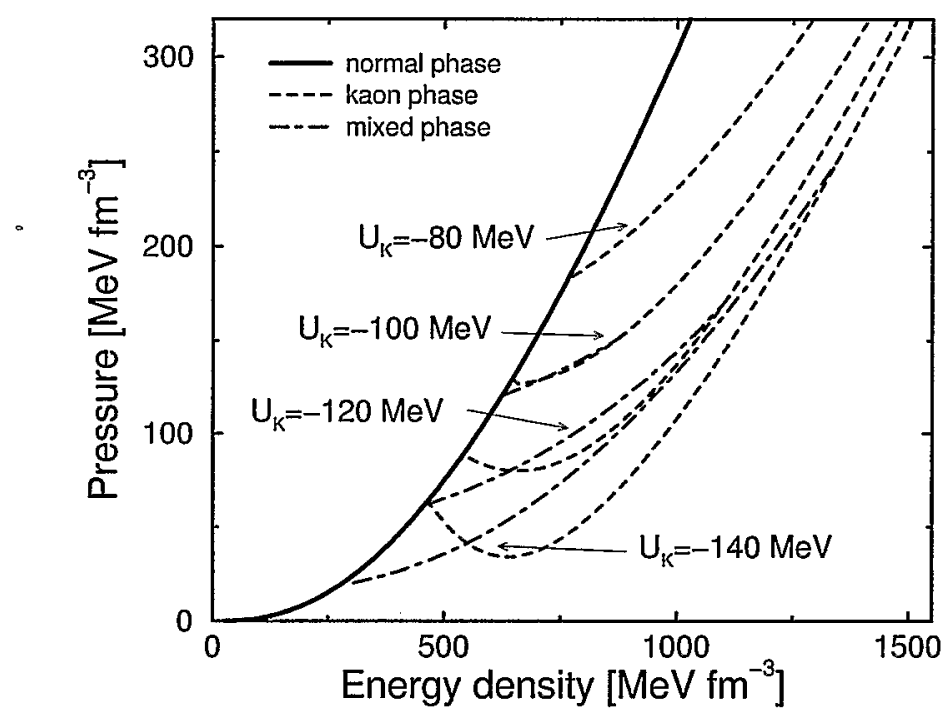

Fig. 10. EoS of kaon condensed matter. The physical solution of the mixed phase is given by the Gibbs construction (dash-dotted lines) as taken from [14]

neutron star matter with a kaon condensate. The pure charge neutral nucleon phase is shown by the solid line. The pure charge neutral kaon condensed phase by the dotted lines. A mechanical instability of the latter phase is apparent for some density range as the slope gets negative, $d p / d \epsilon<0$. The Gibbs construction for the mixed phase, plotted by the dash-dotted lines, is mechanical stable and is a continuously rising function of the density. The mixed phase starts at a lower density compared to the onset of the pure charge neutral kaon condensed phase and can extend to rather large densities. The phase transition turns out to be of second order for small values of the optical potential, i.e. $U_{K}\left(\rho_{0}\right) \geq-80 \mathrm{MeV}$. For both orders of the phase transition, the EoS is considerably softened due to kaon condensation.

If a mixed phase is formed, there is a new degree of freedom to maximize the pressure: the redistribution of charge between the two phases. There are three possible solutions for the charge density: i) the pure nucleon phase with $q_{K}=0$, ii) the pure kaon condensed phase with $q_{N}=0$, and iii) the mixed phase with

$$
q_{\text {total }}=(1-\chi) q_{N}\left(\mu_{B}, \mu_{e}\right)+\chi q_{K}\left(\mu_{B}, \mu_{e}\right)=0,
$$

where $\chi$ is the volume fraction of the two phases. The total global charge is still zero, while the two phases of the mixed phase can have very large local electric (opposite) charge densities.

The charge density in the mixed phase as a function of the volume fraction is plotted in Fig. 11. The nucleon phase starts with zero charge density. Its charge 


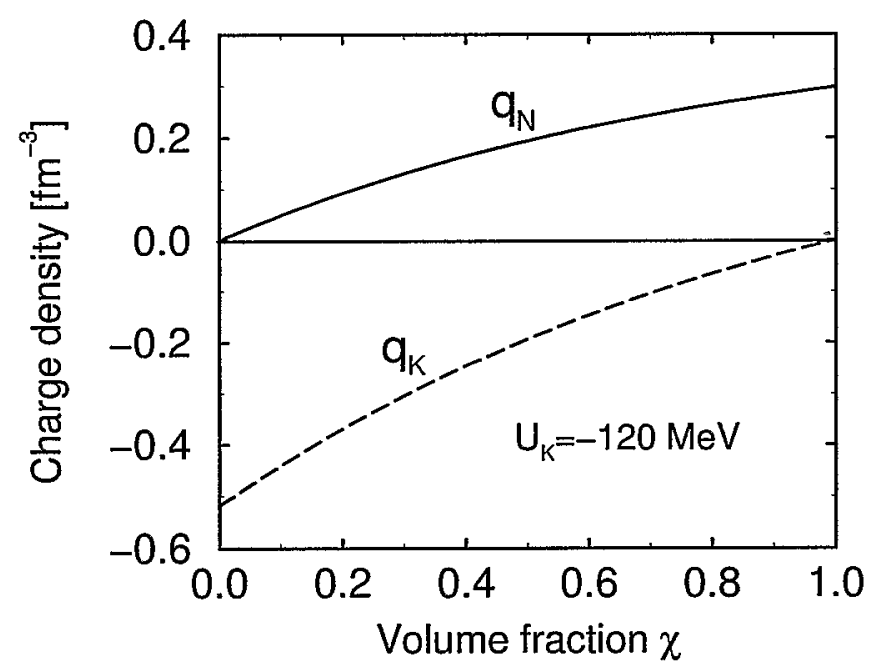

Fig. 11. Charge densities of the two phases in the mixed phase (taken from [14])

density is getting positive in the mixed phase, as it is energetically favoured to have about equal amounts of protons and neutrons in matter. The positive charge of the nucleon phase is compensate by the kaon condensed phase. The latter phase starts at large negative charge density and stops at zero charge density at the end of the mixed phase. For larger density, the pure neutrally charged kaon condensed phase prevails. The charge is distributed between the two phases in the mixed phase, and geometrical (charged) structures appear. These structures are similar to those discussed for the liquid-gas phase transition in the neutron star crust [57] and for the phase transition to deconfined matter [58].

In the selfconsistent approach used here for nucleons and kaons, the crucial ingredience for calculating the geometric structures, the surface tension between the two phases, can be calculated within the model $[59,60]$. The resulting sizes of the geometric structures are summarized in Fig. 12. First, bubbles of the kaon condensed phase appear. Then at larger density, here lower radii, the kaon condensed phase forms rods, then slabs. In the core, the situation reverses and nucleonic slabs form which are immersed in the kaon condensed phase. If one increases the density even further, nucleonic rods, then drops will form ending finally in a pure kaon condensed phase. For the EoS used in Fig. 12, the maximum density reached inside the neutron star is too low to achieve these phases. The size of the structures is around $10 \mathrm{fm}$ and the separation $20-30 \mathrm{fm}$, not far from the size of heavy nuclei of say $7 \mathrm{fm}$. Compared to the size of the neutron star, the mixed phase structures are microscopic and will effect transport and cooling phenomena inside the neutron star (see e.g. [59,61]). 


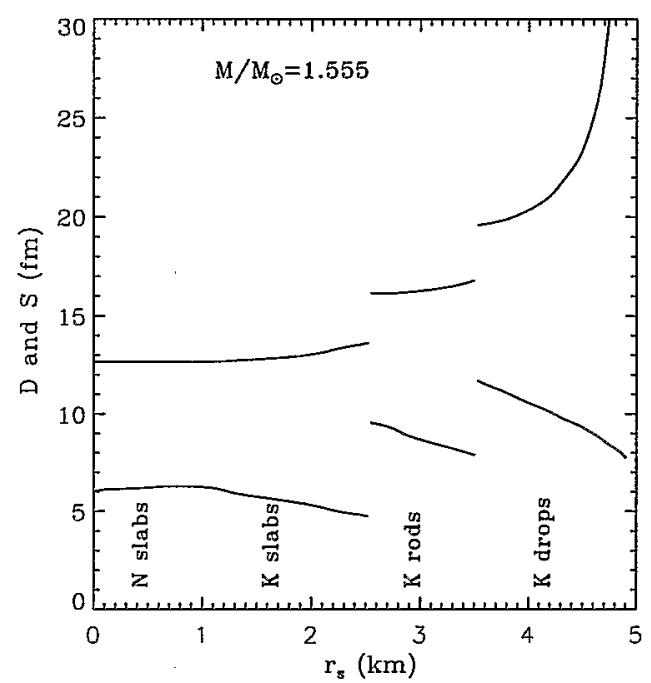

Fig. 12. Sizes of the geometrical structures appearing in the mixed phase (from [60])

One might wonder, why there exists a phase boundary for the nucleons, as they are present in both phases. In the relativistic mean-field model, the mass of the nucleons is shifted in dense matter due to the interaction with the scalar field. In the calculation for the mixed phase, it turns out, that Gibbs conditions are satisfied for different field configurations, i.e. for different values of the effective nucleon masses in the two phases. The difference of the effective nucleon masses is about a factor two in the mixed phase. Hence, the nucleons in the two phases are indeed distinguishable.

The appearance of the kaon condensed phase has certain impacts also on the global properties of the neutron star. As kaon condensation softens the EoS, the maximum mass of neutron stars will be reduced (see e.g. [9]). Figure 13 shows the mass-radius relation for a neutron star with a kaon condensed phase for different strengths of the kaon-nucleon interactions. For moderate attraction for the $\mathrm{K}^{-}$, the maximum mass of a neutron stars drops while the minimum radius increases slightly. For larger attraction, a considerable fraction of the neutron star is in the mixed phase and the maximum mass as well as the minimum radius decreases. For the largest attraction studied here, the minimum radius changes drastically from values of $12 \mathrm{~km}$ without $\mathrm{K}^{-}$condensation to $8 \mathrm{~km}$ with $\mathrm{K}^{-}$ condensation. The maximum mass of the neutron star is lowered from $1.8 \mathrm{M}_{\odot}$ to $1.4 \mathrm{M}_{\odot}$. Note, that there are no instabilities for the Gibbs construction of the mixed phase. The Maxwell construction, shown by dashed lines, is mechanically unstable for some intermediate ranges of the radius. 


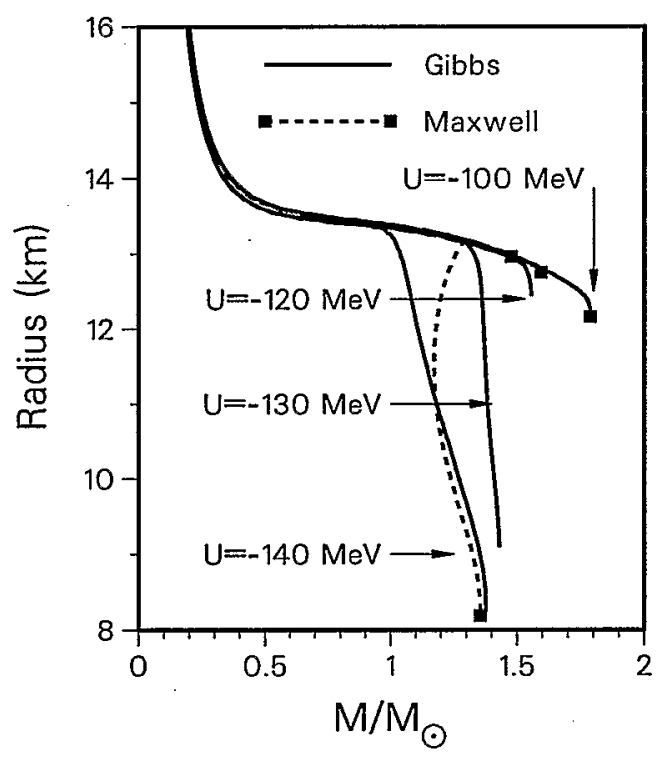

Fig. 13. The mass-radius relation of a neutron star with kaon condensation for different values of the $\mathrm{K}^{-}$optical potential at $\rho_{0}$ (taken from [14])

\subsection{Effects of hyperonization on kaon condensation}

Hyperons may appear around twice normal nuclear density in beta-stable matter (see [56] and references therein). In the last few years, this picture has gained support by various different, modern model calculations. Hyperons (either the $\Lambda$ or the $\Sigma^{-}$) are present in neutron star matter at $2 \rho_{0}$ within effective nonrelativistic potential models [62], the Quark-Meson Coupling Model [63], extended Relativistic Mean-Field approaches [11,12], Relativistic Hartree-Fock [64], BruecknerHartree-Fock [65,66], and chiral effective Lagrangians [67]. Whether the $\Lambda$ or the $\Sigma^{-}$appears first, depends sensitively on the chosen isospin interaction of the $\Sigma$ hyperons. In any case, these two hyperons appear around $(2-4) \rho_{0}$ in the model calculations listed above, which is before kaon condensation sets in.

In most of the modern EoS, the interaction between the baryons is mediated by meson exchange. The nucleon parameters are fitted to properties of nuclei or nuclear matter. Some of the hyperon coupling constants, say the ones to the scalar field, are fixed by the optical potential as extracted from hypernuclear data [68] (see also our discussion in the previous section). The quark model (SU(6) symmetry) can be used to constrain the hyperon coupling constants to the vector fields. The latter choice is often relaxed. While the $A$ coupling constants, and to some extend the ones for the $\Xi$, can be constrained by hypernuclear data, the ones for the $\Sigma$ hyperons can not. Studies of $\Sigma^{-}$atoms indicate a repulsive potential for the $\Sigma$ in nuclei [69]. Another point of uncertainty is related with the interaction between hyperons themselves. Apart from the $\Lambda \Lambda$ interaction, 
nothing is known about the hyperon-hyperon interaction. Only a few calculations have addressed this issue for neutron star matter so far $[12,62,66]$.

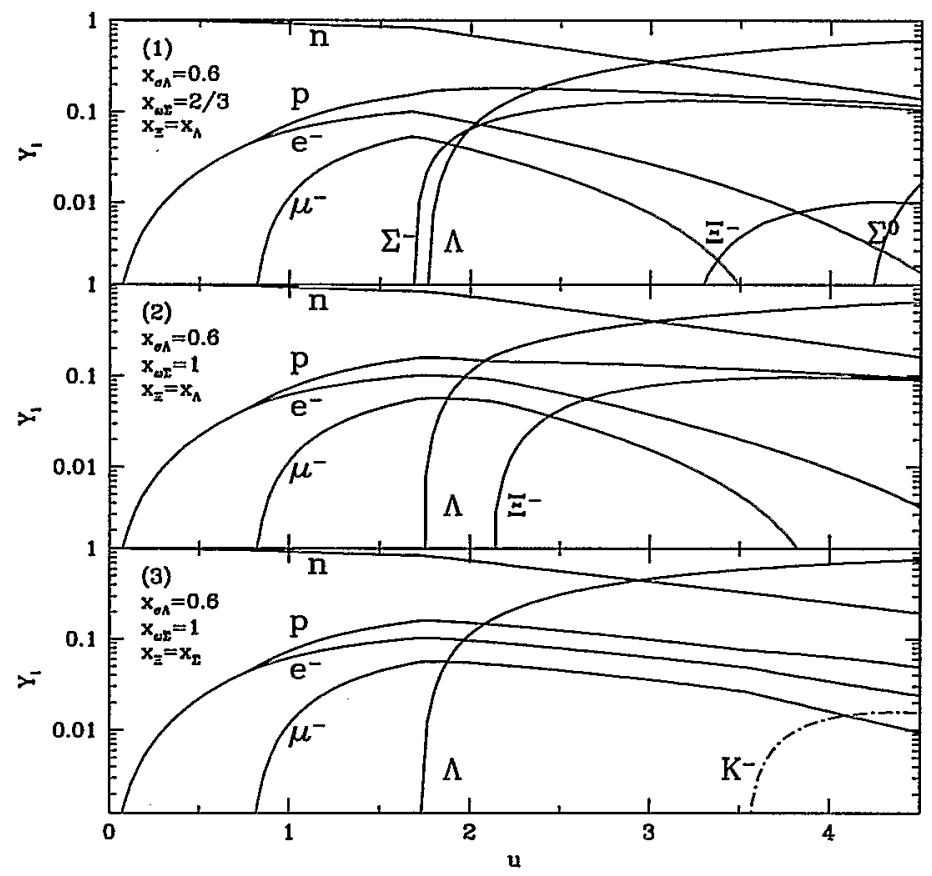

Fig. 14. The particle fractions in neutron star matter versus the relative baryon density $u=\rho / \rho_{0}$. The three panels correspond to different choices for the hyperon coupling constants (from [11])

A representative hyperon population as a function of density is plotted in Fig. 14. The three panels shown corresponds to three different choices of the hyperon vector coupling constants. In the upper panel, the ratio of all hyperon vector coupling constants to the one of the nucleon is set to about $x_{\omega Y}=2 / 3$. This is the ratio predicted by the quark model or $\mathrm{SU}(6)$ symmetry for the coupling constant to the $\omega$ meson for the $\Lambda$ and the $\Sigma$; for the $\Xi$ it would be, of course, only $1 / 3$. The $\Sigma^{-}$and the $\Lambda$ appear at a little bit less than $2 \rho_{0}$. The heavier $\Xi$ hyperon is present in matter at $3.3 \rho_{0}$. If one arbitrarily increases the vector coupling constant of the $\Sigma$ meson to be equal to the one for nucleons, $\boldsymbol{x}_{\omega \Sigma}=1$, the $\Sigma$ hyperons do not appear (see middle panel). The critical density for the onset of the $\Xi^{-}$is then shifted to $2.2 \rho_{0}$, so that the $\Xi^{-}$takes over to some extent the role of the $\Sigma^{-}$. If the corresponding ratio for the $\Xi$ is also increased to $x_{\omega \Xi}=1$, also the $\Xi$ population vanishes (lower panel). The critical density for the $A$ is unchanged. Only for the latter case, a kaon condensed phase emerges at $3.6 \rho_{0}$ in these model calculations. 
Note, that the electron fraction decreases, once hyperons are in the system [56]. This means, that the electron chemical potential does not only saturate but is substantially lowered when hyperons are present in matter. The obvious reason is, that the negative charge needed to cancel the positive charge of the protons is carried now by the negatively charged hyperons instead. Another reason is, that any appearance of a new degree of freedom in matter lowers the overall Fermi momenta of nucleons and leptons, be it charged or not. The latter effect is apparent from the lowest panel of Fig. 14, where just the presence of the neutral $A$ hyperon lowers the electron fraction. The general feature, that hyperons lower the electrochemical potential, has been restressed by Glendenning most recently [70]. As the onset of $\mathrm{K}^{-}$condensation is given by the equality of the effective energy of the $\mathrm{K}^{-}$and the electrochemical potential, it is evident, that the presence of hyperons at least increase the critical density for kaon condensation. Glendenning discussed even before the work of Kaplan and Nelson [5] the destructive effect of hyperons for the appearance of kaon condensation in neutron stars [56]. In more recent works, the in-medium effects for kaons were incorporated in the model calculations including hyperons, and it was found that the onset for kaon condensation is shifted to higher density [11] or does not take place at all [12] (see below).

The hyperonization effects the mass-radius relation for neutron stars. As for any new degree of freedom, the EoS is also softened by hyperons. The maximum possible mass of a neutron star can be lowered by hyperons by about $(0.4-0.7) M_{\odot}[68,11]$. According to the work of [11], kaon condensation without hyperons reduce the maximum mass by only $(0.1-0.2) M_{\odot}$ and the combined effect of kaons and hyperons shift the maximum mass down by about $0.5 M_{\odot}$. So, the main effect in the reduction of the maximum mass stems from the hyperon degree of freedom. We note, that the reduction of the maximum mass due to kaons only is rather model dependent. Other estimates find changes up to $\Delta M=0.4 M_{\odot}$ due to kaon condensation $[9,14]$, but hyperons are ignored in these latter calculations.

There can be an additional hindrance for kaon condensation which is related to the hyperon-hyperon interaction. The vector meson $\phi$ controls the interaction between hyperons at large hyperon densities [12]. The inclusion of the $\phi$ meson models also the kaon-hyperon interaction. As the $\phi$ meson couples solely to strange quarks in $\mathrm{SU}(6)$ symmetry, it is repulsive for hyperons and the $\mathrm{K}^{-}$and attractive for the $\mathrm{K}^{+}$. For neutron star matter with hyperons, the $\phi$ meson exchange can saturate the effective energy of the $\mathrm{K}^{-}$, so that kaon condensation can not happen at all. This effect is depicted in Fig. 15 as a function of density. The effective energy of the $\mathrm{K}^{-}$drops at low density while the one for the $\mathrm{K}^{+}$ increases. At $2 \rho_{0}$, when hyperons are getting populated, the effective energy of the $\mathrm{K}^{-}$saturates as the $\mathrm{K}^{-}$feels the repulsive contribution from the hyperons. On the contrary, the $\mathrm{K}^{+}$energy starts to drop at this density as the $\mathrm{K}^{+}$-hyperon interaction is attractive. The lower curves show the electrochemical potential which has to be crossed by the effective $\mathrm{K}^{-}$energy for the $\mathrm{K}^{-}$to be present. As the electrochemical potential as well as the $\mathrm{K}^{-}$effective energy saturate at large 


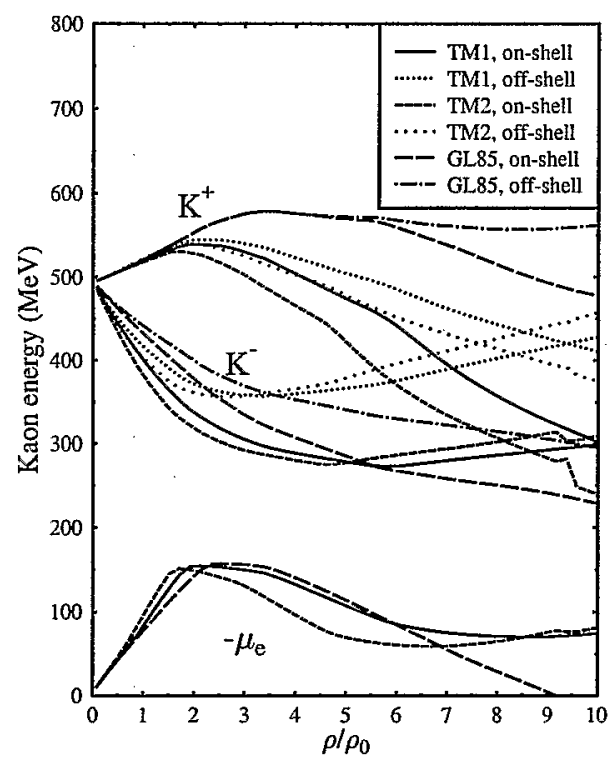

Fig. 15. The effective energy of kaons as well as the electrochemical potential versus the baryon density for neutron star matter (taken from [12])

density due to the presence of hyperons, that crossing does not happen and kaon condensation is prevented in this scenario.

\section{Summary and Outlook}

We have discussed the elementary kaon-nucleon interaction as derived from scattering data and the in-medium changes of the $\mathrm{K}^{-}$as deduced from kaonic atoms. Various coupled channel calculations using chiral effective interactions or the boson exchange model with higher order partial waves and their results for the $\mathrm{K}^{-}$ properties in dense matter have been reviewed. The extracted range of the optical potential has been used to study the phase transition to kaon condensation in neutron star matter. We outlined the effects of a first order phase transition due to the presence of structures in the mixed phase. Consequences for the global properties of neutron stars, i.e. a reduced maximum mass and minimum radius, have been addressed. Finally, we examined the rôle of hyperons on the onset of kaon condensation in neutron stars.

It is clear from our, review, that the discussion about kaon condensation is far from being complete at present. The topic is a challenge both for experimentalists as well as for theorists, demonstrating a growing interplay between traditional nuclear physics, heavy-ion physics, and astrophysics. For example, the importance of $\mathrm{p}$-wave interactions also for cold, dense neutron star matter 
has been stressed, but further work is needed in that direction. Especially, the precise value of the optical potential of the $\mathrm{K}^{-}$is a crucial ingredience for the neutron star matter calculation and needs to be pinned down more precisely, be it by the experimental study of deep lying levels in kaonic atoms, subthreshold kaon production in heavy-ion collisions at GSI, or by the mass-radius measurement of neutron stars.

The inclusion of hyperons for the equation of state is certainly a necessary one, but there are only a few works which have been devoted to this issue. In particular, the hyperon-hyperon interaction as well as the kaon-hyperon interaction can be important for the onset of kaon condensation and could be addressed by e.g. a chiral SU(3) symmetric model. A consistent calculation, incorporating realistic kaon-baryon as well as a baryon-baryon interactions on the same basis is still missing.

Last but not least, we point out, that kaon condensation can have impacts on other facets of neutron stars, like the evolution of proto-neutron stars and the deconfinement phase transition to quark matter. Concerning the latter point, the appearance of both, a kaon condensed phase and a quark matter phase, will lead to a triple point of strongly interacting matter inside a neutron star. At this point, the three phases, the normal hadronic, the kaon condensed and the quark matter phase, are in equilibrium. Beyond that point, the kaon condensed phase and the quark matter phase are forming a mixed phase. The presence of this triple point might have interesting implications for transport phenomena in neutron stars.

\section{Acknowledgments}

We thank all colleagues that have collaborated with us in obtaining the results presented here, especially M.B. Christiansen, M. Effenberger, N.K. Glendenning, S. Hirenzaki, V. Koch, T.T.S. Kuo, I.N. Mishustin, Y. Okumura, E. Oset, A. Polls, H. Toki, L. Tolos. AR acknowledges support by the DGICYT project PB98-1247, the Generalitat de Catalunya Grant 1998SGR-11 and the EU contract FMRX-CT98-0169. JSB thanks RIKEN, BNL and the U.S. Department of Energy for providing the facilities essential for the completion of this work. JW thanks the Gesellschaft für Schwerionenforschung (GSI), Darmstadt, and the Bundesministerium für. Bildung nnd Forschung (BMBF) for their support.

\section{U.S.D.O.E.-DE-ACO2-98CH10886 \\ References}

1. T. Schäfer and E.V. Shuryak, Rev. Mod. Phys. 70, 323 (1998).

2. J. Gasser and H. Leutwyler, Ann. Phys. (NY) 158, 142 (1984); Nucl. Phys. B250, 465 (1985).

3. N. Kaiser, P.B. Siegel and W. Weise, Nucl. Phys. A594, 325 (1995).

4. N. Kaiser, T. Waas and W. Weise, Nucl. Phys. A612, 297 (1997).

5. D.B. Kaplan and A.E. Nelson, Phys. Lett. B 175, 57 (1986), ibid 179, 409 (Erratum). 
6. G.E. Brown, K. Kubodera, M. Rho, and V. Thorsson, Phys. Lett. B 291, 355 (1992).

7. V. Thorsson, M. Prakash, and J.M. Lattimer, Nucl. Phys. A572, 693 (1994).

8. H. Fujii, T. Maruyama, T. Muto, and T. Tatsumi, Nucl. Phys. A597, 645 (1996).

9. G.Q. Li, C.-H. Lee, and G.E. Brown, Phys. Rev. Lett. 79, 5214 (1997).

10. P.J. Ellis, R. Knorren, and M. Prakash, Phys. Lett. B 349, 11 (1995).

11. R. Knorren, M. Prakash, and P.J. Ellis, Phys. Rev. C 52, 3470 (1995).

12. J. Schaffner and I.N. Mishustin, Phys. Rev. C 53, 1416 (1996).

13. N.K. Glendenning and J. Schaffner-Bielich, Phys. Rev. Lett. 81, 4564 (1998).

14. N.K. Glendenning and J. Schaffner-Bielich, Phys. Rev. C 60, 025803 (1999).

15. T. Tatsumi and M. Yasuhira, Phys. Lett. B 441, 9 (1998); T. Tatsumi and M. Yasuhira, Nucl. Phys. A653, 133 (1999).

16. E. Friedman, A. Gal, and C.J. Batty, Nucl. Phys. A579, 518 (1994).

17. R. Barth et al., Phys. Rev. Lett. 78, 4007 (1997); F. Laue et al., Phys. Rev. Lett. 82, 1640 (1999).

18. W. Cassing, E.L. Bratkovskaya, U. Mosel, S. Teis, and A. Sibirtsev, Nucl. Phys. A614, 415 (1997); E.L. Bratkovskaya, W. Cassing, and U. Mosel, Nucl. Phys. A622, 593 (1997).

19. G.Q. Li, C.M. Ko, and X.S. Fang, Phys. Lett. B 329, 149 (1994).

20. G.Q. Li and C.M. Ko, Phys. Rev. C 54, 2159 (1996).

21. G.Q. Li, C.-H. Lee, and G.E. Brown, Nucl. Phys. A625, 372 (1997).

22. J. Schaffner-Bielich, V. Koch, and M. Effenberg, Nucl. Phys. A669, 153 (2000).

23. A. Sibirtsev and W. Cassing; Nucl. Phys. A641, 476 (1998).

24. C.-H. Lee, G.E. Brown, and M. Rho, Phys. Lett. B 335, 266 (1994); C.-H. Lee, G.E. Brown, D.P. Min, and M. Rho, Nucl. Phys. A585, 401 (1995).

25. C.-H. Lee, Phys. Rep. 275, 255 (1996).

26. G. Mao, P. Papazoglou, S. Hofmann, S. Schramm, H. Stöcker, and W. Greiner, Phys. Rev. C 59, 3381 (1999).

27. J. Schaffner-Bielich, I.N. Mishustin, and J. Bondorf, Nucl. Phys. A625, 325 (1997).

28. K. Tsushima, K. Saito, A.W. Thomas, and S.V. Wright, Phys. Lett. B 429, 239 (1998).

29. M. Alberg, E.M. Henley, and L. Wilets, Ann. Phys. (N.Y.) 96, 43 (1976).

30. L.R. Staronski and S. Wycech, J. Phys. G13, 1361 (1987).

31. V. Koch, Phys. Lett. B 337, 7 (1994).

32. T. Waas, N. Kaiser, and W. Weise, Phys. Lett. B 365, 12 (1996).

33. T. Waas and W. Weise, Nucl. Phys. A625, 287 (1997).

34. M. Lutz, Phys. Lett. B 426, '12 (1998).

35. A. Ramos and E. Oset, Nucl. Phys. A671, 481 (2000).

36. E. Oset and A. Ramos, Nucl. Phys. A635, 99 (1998).

37. J. A. Oller and E. Oset, Nucl. Phys. A620, 438 (1997); erratum Nucl. Phys. A624, 407 (1999).

38. J. A. Oller, E. Oset, and J. R. Peláez, Phys. Rev. Lett. 80, 3452 (1998); Phys. Rev. D 59, 074001 (1999); erratum Phys. Rev. D 60, 099906 (1999).

39. J.A. Oller, E. Oset, and A. Ramos, Prog. Part. Nucl. Phys. 45, 157 (2000).

40. L. Tolós, A. Ramos, A. Polls, and T.T.S. Kuo, nucl-th/0007042

41. A. Müller-Groeling, K. Holinde, and J. Speth, Nucl. Phys. A513, 557 (1990).

42. H. Bandō, T. Motoba, and J. Žofka, Int. J. Mod. Phys. A5, 4021 (1990).

43. C.J. Batty et al., Phys. Lett: 74B, 27 (1978); C.J. Batty et al., Phys. Lett. 87B, 324 (1979).

44. E. Oset, P. Fernández de Córdoba, L.L. Salcedo, and R. Brockmann, Phys. Rep. 188, 79 (1990). 
45. C.J. Batty, E. Friedman, and A. Gal, Phys. Lett. B 335, 273 (1994).

46. J. Caro, N. Kaiser, S. Wetzel, and W. Weise, Nucl. Phys. A672, 249 (2000).

47. A. Ramos, E. Oset, and L.L. Salcedo, Phys. Rev. C 50, 2314 (1994).

48. S. Hirenzaki, Y. Okumura, H. Toki, E. Oset, and A. Ramos, Phys. Rev. C 61, 055205 (2000).

49. C. J. Batty, E. Friedman, and A. Gal, Phys. Rep. 287, 385 (1997).

50. E. Friedman, A. Gal, J. Mareš, and A. Cieplý, Phys. Rev. C 60, 024314 (1999).

51. C.J. Batty, Nucl. Phys. A372, 418 (1981).

52. A. Baca, C. García-Recio, and J. Nieves, Nucl. Phys. A673, 335 (2000).

53. E. Friedman and A. Gal, Phys. Lett. B 459, 43 (1999).

54. E. Friedman and A. Gal, Nucl. Phys. A658, 345 (1999).

55. T. Kishimoto, Phys. Rev. Lett. 83, 4701. (1999).

56. N.K. Glendenning, Astrophys. J. 293, 470 (1985).

57. D.G. Ravenhall, C.J. Pethick, and J.R. Wilson, Phys. Rev. Lett. 50, 2066 (1983).

58. N.K. Glendenning, Phys. Rev. D 46, 1274 (1992).

59. S. Reddy, G. Bertsch, and M. Prakash, Phys. Lett. B 475, 1 (2000); T. Norsen and S. Reddy, nucl-th/0010075 (2000).

60. M.B. Christiansen, N.K. Glendenning, and J. Schaffner-Bielich, Phys. Rev. C 62, 025804 (2000).

61. J.A. Pons, S. Reddy, P.J. Ellis, M. Prakash and J.M. Lattimer, Phys. Rev. C 62, $035803(2000)$

62. S. Balberg and A. Gal, Nucl. Phys. A625, 435 (1997).

63. S. Pal, M. Hanauske, I. Zakout, H. Stöcker, and W. Greiner, Phys. Rev. C 60, 015802 (1999).

64. H. Huber, F. Weber, M.K. Weigel, and C. Schaab, Int. J. Mod. Phys. E7, 301 (1998).

65. M. Baldo, G.F. Burgio, and H.J. Schulze, Phys. Rev. C 61, 055801 (2000).

66. I. Vidana, A. Polls, A. Ramos, L. Engvik, and M. Hjorth-Jensen, Phys. Rev. C 62, 035801 (2000).

67. M. Hanauske, D. Zschiesche, S. Pal, S. Schramm, H. Stöcker, and W. Greiner, Astrophys. J. 537, 50320 (2000).

68. N.K. Glendenning and S.A. Moszkowski, Phys. Rev. Lett. 67, 2414 (1991).

69. J. Mares, E. Friedman, A. Gal, and B.K. Jennings, Nucl. Phys. A594; 311 (1995).

70. N.K. Glendenning, nucl-th/0009082 (2000). 